\title{
A Rickettsiella Endosymbiont Is a Potential Source of Essential B-Vitamins for the Poultry Red Mite, Dermanyssus gallinae
}

\author{
Daniel R. G. Price ${ }^{1 *}$, Kathryn Bartley ${ }^{1}$, Damer P. Blake ${ }^{2}$, Eleanor Karp-Tatham ${ }^{2}$, \\ Francesca Nunn ${ }^{1}$, Stewart T. G. Burgess ${ }^{1}$ and Alasdair J. Nisbet ${ }^{1}$ \\ ${ }^{1}$ Moredun Research Institute, Penicuik, United Kingdom, ${ }^{2}$ Department of Pathobiology and Population Sciences, \\ Royal Veterinary College, London, United Kingdom
}

\section{OPEN ACCESS}

Edited by:

Yuval Gottlieb,

Hebrew University of Jerusalem,

Israel

Reviewed by:

Jen White,

University of Kentucky, United States

Joshua Benoit,

University of Cincinnati, United States

*Correspondence:

Daniel R. G. Price

daniel.price@moredun.ac.uk

Specialty section: This article was submitted to Microbial Symbioses,

a section of the journal

Frontiers in Microbiology

Received: 14 April 2021

Accepted: 28 July 2021

Published: 03 September 2021

Citation:

Price DRG, Bartley K, Blake DP,

Karp-Tatham E, Nunn F,

Burgess STG and Nisbet AJ (2021)

A Rickettsiella Endosymbiont Is a

Potential Source of Essential

B-Vitamins for the Poultry Red Mite,

Dermanyssus gallinae.

Front. Microbiol. 12:695346.

doi: 10.3389/fmicb.2021.695346
Many obligate blood-sucking arthropods rely on symbiotic bacteria to provision essential $B$ vitamins that are either missing or at sub-optimal levels in their nutritionally challenging blood diet. The poultry red mite Dermanyssus gallinae, an obligate blood-feeding ectoparasite, is a serious threat to the hen egg industry. Poultry red mite infestation has a major impact on hen health and welfare and causes a significant reduction in both egg quality and production. Thus far, the identity and biological role of nutrient provisioning bacterial mutualists from $D$. gallinae are little understood. Here, we demonstrate that an obligate intracellular bacterium of the Rickettsiella genus is detected in $D$. gallinae mites collected from 63 sites (from 15 countries) across Europe. In addition, we report the genome sequence of Rickettsiella from D. gallinae (Rickettsiella - D. gallinae endosymbiont; Rickettsiella DGE). Rickettsiella DGE has a circular 1.89 Mbp genome that encodes 1,973 proteins. Phylogenetic analysis confirms the placement of Rickettsiella DGE within the Rickettsiella genus, related to a facultative endosymbiont from the pea aphid and Coxiellalike endosymbionts (CLEs) from blood feeding ticks. Analysis of the Rickettsiella DGE genome reveals that many protein-coding sequences are either pseudogenized or lost, but Rickettsiella DGE has retained several B vitamin biosynthesis pathways, suggesting the importance of these pathways in evolution of a nutritional symbiosis with $D$. gallinae. In silico metabolic pathway reconstruction revealed that Rickettsiella DGE is unable to synthesize protein amino acids and, therefore, amino acids are potentially provisioned by the host. In contrast, Rickettsiella DGE retains biosynthetic pathways for B vitamins: thiamine (vitamin B1) via the salvage pathway; riboflavin (vitamin B2) and pyridoxine (vitamin B6) and the cofactors: flavin adenine dinucleotide (FAD) and coenzyme A (CoA) that likely provision these nutrients to the host.

Keywords: endosymbiont, mutualist, Gammaproteobacteria, vitamin biosynthesis, hematophagous, microbiome 


\section{INTRODUCTION}

Animals live in a diverse bacterial world and mutualistic associations with bacteria can provide these animals with novel biochemical traits to exploit an otherwise inaccessible ecological niche (McFallNgai et al., 2013). For example, specialist phloem-feeding insects of the order Hemiptera depend on bacterial endosymbionts to synthesize and provide essential amino acids that are largely absent in their phloem sap diet (Moran, 2007). Similarly, obligate bloodfeeding arthropods, including insects, ticks, and mites associate with nutritional mutualists that provide essential vitamins and cofactors that are in limited supply from their blood diet (recently reviewed in Husnik, 2018). Typically, as a result of relaxed selection, the genomes of nutritional mutualists are reduced relative to their free-living ancestors. Genes that are essential for symbiosis are retained, while non-essential genes are lost, resulting in small compact genomes (as reviewed by Toft and Andersson, 2010; McCutcheon and Moran, 2012). The microbiome of obligate blood-feeding invertebrates is often dominated by a single $\mathrm{B}$ vitamin provisioning symbiont. For example, the blood-feeding African soft tick (Ornithodoros moubata) is associated with a Francisella (strain F-Om) that provides the host with essential B vitamins to supplement its blood meal diet (Duron et al., 2018). The genome sequence of Francisella F-Om bears the hallmarks of a host-adapted bacterial endosymbiont, with dramatic genome reduction resulting from loss of redundant genes that are not required for a symbiotic function. Importantly, Francisella F-Om retains biosynthesis pathways for B vitamins biotin (B7), riboflavin (B2), folic acid (B9) and cofactors coenzyme A (CoA) and flavin adenine dinucleotide (FAD) to supplement deficiencies in the hosts diet (Duron et al., 2018). This pattern of genome reduction and retention of $\mathrm{B}$ vitamin biosynthesis pathways is also observed in Coxiella-like endosymbionts (CLEs) from obligate blood-feeding ticks. Recent genome sequence studies revealed that, in comparison to the pathogen Coxiella burnetii (genome size 2.03 Mbp), CLEs from ticks have reduced genomes, as small as $0.66 \mathrm{Mbp}$ for CLE from the lone star tick (CLE of Amblyomma americanum). Yet, these nutritional mutualists retain pathways for $\mathrm{B}$ vitamin and cofactor biosynthesis to supplement the nutritional requirements of their blood-feeding host (Smith et al., 2015).

The poultry red mite (Dermanyssus gallinae) is an obligate blood-feeding ectoparasite that feeds on avian blood. This mite has a worldwide distribution and is endemic in many commercial poultry farms, with up to $83 \%$ of European egg-laying facilities infested by D. gallinae (George et al., 2015). Infestation of poultry houses has a serious impact on hen health and welfare and causes a significant reduction in both egg quality and production. Infestations can reach up to 500,000 mites per bird and cause welfare issues, including anemia, irritation, and even death of hens by exsanguination (Sigognault Flochlay et al., 2017). In the EU, D. gallinae infestation costs the poultry industry in excess of $€ 231$ million per annum due to production losses alone (Sigognault Flochlay et al., 2017). In addition, D. gallinae has been implicated in the transmission of avian viral and bacterial disease (Huong et al., 2014; Sigognault Flochlay et al., 2017).

To utilize blood as a food source, our current hypothesis is that D. gallinae associates with bacterial mutualists, which synthesize and supply essential B vitamins and cofactors that are absent in the blood diet of the mite. A previous microbiome analysis of $D$. gallinae demonstrates that adult female mites have a simple microbiome, with 10 operational taxonomic units (OTUs) accounting for between 90 and 99\% of the observed microbial diversity (Hubert et al., 2017). Furthermore, only four bacterial taxa, including: Bartonella, Cardinium, Wolbachia, and Rickettsiella, were present across all D. gallinae life-stages (Hubert et al., 2017). Data presented here, based on bacterial $16 \mathrm{~S}$ rRNA amplicon sequencing confirms the presence of Rickettsiella in D. gallinae eggs, in agreement with previous studies (De Luna et al., 2009; Hubert et al., 2017). Here, we investigate the distribution of the previously identified Rickettsiella - D. gallinae endosymbiont (Rickettsiella DGE; Hubert et al., 2017) in D. gallinae across Europe, determine the complete genome of Rickettsiella DGE and examine this genome for evidence of biosynthesis pathways, which would supplement the diet of its host, D. gallinae.

\section{MATERIALS AND METHODS}

\section{Mite Collection and Endosymbiont- Enriched DNA Preparation}

Dermanyssus gallinae were collected from a single commercial laying hen facility in the Scottish Borders, United Kingdom and maintained in $75 \mathrm{~cm}^{2}$ canted tissue culture flasks (Corning Inc., Corning, NY, United States) at $4^{\circ} \mathrm{C}$ for up to 4 weeks after collection. For experiments requiring mite eggs, freshly collected mixed stage and gender mites were placed into vented $25 \mathrm{ml}$ Sterilin universal tubes and maintained at $25^{\circ} \mathrm{C}, 75 \%$ relative humidity in a Sanyo MLR-350H incubator and eggs were collected the following day.

Since obligate bacterial endosymbionts are uncultivable outside the host, bacteria were derived from D. gallinae tissue lysates and host cells were removed from the extract using host depletion solution (Zymo Research, Irvine, CA, United States). Briefly, live mixed life-stage mites were surface sterilized with $70 \%(v / v)$ ethanol for $30 \mathrm{~s}$ at room temperature followed by three $1 \mathrm{~min}$ washes in nuclease-free water. Mites (approx. $25 \mathrm{mg}$ ) were then homogenized in $200 \mu \mathrm{l}$ nuclease-free water using a tube pestle and host cells lysed by addition of $1 \mathrm{ml}$ of host depletion solution (Zymo Research, Irvine, CA, United States) with a $15 \mathrm{~min}$ incubation at room temperature with end over end mixing. Intact bacterial cells were pelleted by centrifugation at $10,000 \times g$ for $5 \mathrm{~min}$ at room temperature and DNA extracted from the pellet using a DNeasy ${ }^{\circledR}$ Blood \& Tissue kit (Qiagen, Hilden, Germany). DNA concentration was assessed by the Qubit $^{\mathrm{TM}}$ dsDNA BR Assay Kit (Thermo Fisher Scientific, Waltham, MA, United States) and $1 \%(w / v)$ agarose/TAE gel electrophoresis.

\section{S rRNA Amplicon Sequencing and Classification}

Poultry red mite eggs were collected as described above using mites from the same commercial laying hen facility as described in section "Mite Collection and Endosymbiont-Enriched DNA 
Preparation." Mite eggs were surface sterilized by two $5 \mathrm{~min}$ washes in $0.1 \%(w / v)$ benzalkonium chloride followed by two $5 \mathrm{~min}$ washes in $70 \%(v / v)$ ethanol. DNA was extracted from eggs using a DNeasy ${ }^{\circledR}$ Blood \& Tissue kit (Qiagen, Hilden, Germany) with a lysozyme pre-treatment to lyse bacterial cells. DNA was quantified using a NanoDrop ${ }^{\mathrm{TM}}$ One spectrophotometer (Thermo Fisher Scientific, Waltham, MA, United States) and DNA molecular weight determined on a $1 \%(w / v)$ agarose/ TAE gel. A reagent-only control DNA extraction was performed in parallel using the same DNA extraction kit.

The presence of bacterial DNA in mite eggs was verified by PCR using universal bacterial $16 \mathrm{~S}$ rRNA gene primers 27F-short (5'-GAGTTTGATCCTGGCTCA-3') and 1507R (5'-TACCTTGTTACGACTTCACCCCAG-3'). Each $50 \mu \mathrm{l}$ PCR reaction contained template DNA $(100 \mathrm{ng}), 1 \mathrm{U}$ Platinum ${ }^{\mathrm{TM}}$ Taq DNA Polymerase (Thermo Fisher Scientific, Waltham, MA, United States), $1 \times$ PCR buffer, $1.5 \mathrm{mM} \mathrm{MgCl}_{2}, 0.2 \mathrm{mM}$ of each $\mathrm{dNTP}$ and each primer at $0.2 \mu \mathrm{M}$. Cycling conditions were as follows: $94^{\circ} \mathrm{C}$ for $2 \mathrm{~min}$; 30 cycles of $94^{\circ} \mathrm{C} 30 \mathrm{~s}, 58^{\circ} \mathrm{C} 30 \mathrm{~s}$, $72^{\circ} \mathrm{C} 1 \mathrm{~min} 30 \mathrm{~s}$, and a final hold of $72^{\circ} \mathrm{C}$ for $10 \mathrm{~min}$. A control PCR reaction was performed using the same conditions with an equivalent volume of eluate from the reagent-only control extraction. PCR products were cloned into pJET1.2 using the CloneJet PCR cloning kit (Thermo Fisher Scientific, Waltham, MA, United States) and transformed into chemically competent JM109 Escherichia coli cells (Promega, Madison, WI, United States). Transformants were selected on Lysogeny broth (LB) agar plates containing $100 \mu \mathrm{g} / \mathrm{ml}$ ampicillin at $37^{\circ} \mathrm{C}$. Colony PCR was performed on randomly selected individual colonies using pJET1.2-F (5'-CGACTCACTATAGGGAGAGCGGC-3') and pJET1.2-R (5'-AAGAACATCGATTTTCCATGGCAG-3') vector primers using the previously detailed cycling conditions, except the primer annealing temperature was reduced to $56^{\circ} \mathrm{C}$. PCR products were analyzed on a $1 \%(w / v)$ agarose/TAE gel and colonies containing the expected size amplification product were grown overnight in $10 \mathrm{ml} \mathrm{LB}$ containing $100 \mu \mathrm{g} / \mathrm{ml}$ ampicillin at $37^{\circ} \mathrm{C}$ with shaking at $200 \mathrm{rpm}$. Plasmid DNA was isolated from each clone using Wizard ${ }^{\circledR}$ Plus SV Miniprep kit (Promega, Madison, WI, United States) and a total of 72 individual clones were sequenced with pJET1.2-F and pJET1.2-R primers at Eurofins Genomics Germany $\mathrm{GmbH}$.

To assess the geographical association between D. gallinae and Rickettsiella, we used DNA from a previously published mite collection from 63 sites across Europe (Karp-Tatham et al., 2020). For each collection site, DNA extracted from a single mite was screened by PCR for the presence of Rickettsiella. Diagnostic Rickettsiella primers Rick-F (5'-GTCGAACGGC AGCACGGTAAAGACT-3') and Rick-R (5'-TCGGTTACCTT TCTTCCCCACCTAA-3') were designed based on Rickettsiella specific 16S rRNA regions using alignments in the PhylOPDb database (Jaziri et al., 2014). These primers were designed to amplify a 408 bp fragment of the Rickettsiella 16S rRNA gene. The diagnostic Rickettsiella 16S rRNA PCR primers were checked for specificity by running in silico searches against the current RDP 16S rRNA database (Wang et al., 2007). In addition, the specificity of the primers was validated by PCR on DNA isolated from adult female D. gallinae mites. Amplification products were analyzed by on a $1 \%(w / v)$ agarose/TAE gel to check for size and sequenced, confirming Rickettsiella specific amplification.

For screening European mite DNA, each $25 \mu \mathrm{l}$ PCR reaction contained template DNA (5 ng), 0.5 U Phusion ${ }^{\mathrm{TM}}$ High-Fidelity DNA Polymerase (Thermo Fisher Scientific, Waltham, MA, United States), $1 \times$ PCR buffer, $0.2 \mathrm{mM}$ of each dNTP and each primer at $0.5 \mu \mathrm{M}$. Cycling conditions were as follows: $98^{\circ} \mathrm{C}$ for $30 \mathrm{~s} ; 30$ cycles of $98^{\circ} \mathrm{C} 10 \mathrm{~s}, 68^{\circ} \mathrm{C} 30 \mathrm{~s}, 72^{\circ} \mathrm{C} 30 \mathrm{~s}$, and a final hold of $72^{\circ} \mathrm{C}$ for $10 \mathrm{~min}$. All PCR products were analyzed on a $1 \%(w / v)$ agarose/TAE gel and sequenced in both directions using Rick-seq-F (5'-AACGGCAGCACG GTAAAGAC-3') and Rick-seq-R (5'-AGTGCTTTACAACCC GAAGG-3') primers at Eurofins Genomics Germany $\mathrm{GmbH}$.

16S rRNA sequences were classified with the RDP Classifier 2.13 (training set No. 18; Wang et al., 2007) and sequences with $<80 \%$ bootstrap support as their genus assignment were removed from the dataset. All remaining sequences were used in blastn searches against the GenBank database to identify their top hit.

\section{Genome Sequencing and Assembly}

For the Rickettsiella genome assembly, we used PacBio reads from $D$. gallinae eggs that were generated in a previous study (Burgess et al., 2018). Sequence reads were derived from adult female mites collected at the same commercial laying hen facility as described in section "Mite Collection and Endosymbiont-Enriched DNA Preparation." The data set contained 7,318,092 reads for a total of $63,984,748,667$ bases. Raw reads were mapped against the $D$. gallinae reference genome using Minimap2 v.2.17 (Li, 2018) and unmapped reads were extracted from the resulting BAM files using SAMtools v1.11 (Li et al., 2009). Unmapped reads (814,785 reads for a total of $1,274,422,647$ bases) were assembled using the metaFlye assembler v.2.8.2 under default settings using the --pacbio-raw and --meta flags (Kolmogorov et al., 2020). The assembly containing 652 contigs was visualized with Bandage (Wick et al., 2015), which allowed identification of a circular $1.89 \mathrm{Mbp}$ Rickettsiella genome with $12 \times$ coverage.

For massive parallel sequencing (MPS) host-depleted gDNA was extracted from mixed life-stage mites, D. gallinae mites collected from a commercial egg laying facility, as described in section "Mite Collection and Endosymbiont-Enriched DNA Preparation." DNA was fragmented using a Covaris system, size-selected for 200-400 bp fragments and used to construct a single strand DNA circle library. The library was amplified using phi29 DNA polymerase by rolling circle amplification to make DNA nanoballs (DNBs) and sequenced on a DNBSEQG50 platform as $150 \mathrm{bp}$ paired end reads. Library construction and sequencing were performed by BGI Genomics (Shenzhen, China). This sequencing effort resulted in generation of $174,890,018$ reads for a total of $26,233,502,700$ bases. The reads were used to polish the Rickettsiella consensus sequence. Briefly, short-reads were mapped to the Rickettsiella genome using BWA-MEM aligner v0.7.17 (Li, 2013) and base calls were corrected using five iterative rounds of polishing with Pilon v1.23 (Walker et al., 2014). 
The resultant assembly consisted of a single circular chromosome of $1,888,715 \mathrm{bp}$ with $3,712 \times$ coverage.

\section{Genome Annotation}

The genome was annotated using Prokka v.1.14.6 (Seemann, 2014) and the automated pipeline included coding region prediction by Prodigal (Hyatt et al., 2010) and annotation of non-coding rRNAs using Barrnap and tRNAs using ARAGORN (Laslett and Canback, 2004). As part of the Prokka pipeline, insertion sequences (IS) were annotated using the ISfinder database (Siguier et al., 2006). Candidate pseudogenes were identified based on the length ratios of each predicted Rickettsiella DGE protein against their top blastp hit from searches against the NCBI nr protein database. Rickettsiella DGE proteins that deviated by $+/-25 \%$ compared to their top blastp hit were flagged as potential pseudogenes. Metabolic pathways for amino acids, B vitamins and cofactors were manually inspected using KEGG (Kanehisa and Goto, 2000) and MetaCyc (Caspi et al., 2006) reference pathways. The absence of genes in pathways was verified by tblastn searches against the Rickettsiella genome. The Rickettsiella DGE genome plot was generated using DNAplotter (Carver et al., 2009). Synteny analysis was performed between the Rickettsiella DGE and Rickettsiella viridis genome (accession number: AP018005) using MUMmer (Delcher et al., 2002; nucmer --maxgap =1,000 -mumreference -c 100).

\section{Phylogenetic Analysis}

A phylogenetic relationship of Rickettsiella isolates was reconstructed using $16 \mathrm{~S}$ rRNA sequences obtained from $D$. gallinae. To reconstruct the Rickettsiella phylogeny, we retrieved additional 16S rRNA sequences from GenBank based on sequence similarity to the Rickettsiella DGE 16 S sequence and more distantly related bacteria. This dataset included sequences from Rickettsiella found in various tick species, insect species, and other arthropods. Using this dataset 16S rRNA sequences were aligned using ClustalW (1,013 bp unambiguously aligned sites) and a maximum-likelihood (ML) phylogenetic tree constructed using the Kimura 2-parameter (K2) model with gamma distributed with invariant sites $(\mathrm{G}+\mathrm{I})$. The substitution model was selected based on BIC score (Bayesian Information Criterion) and reliability of the tree was tested using bootstrap analysis (1,000 replicates) with bootstrap values indicated on the tree. All phylogenetic analyzes were performed using MEGA version X (Kumar et al., 2018).

\section{RESULTS AND DISCUSSION}

\section{Rickettsiella Is Present in Dermanyssus gallinae Eggs}

16S rRNA amplicon sequencing of DNA isolated from a pool of surface-sterilized D. gallinae eggs reveals that Rickettsiella is detectable in mite eggs (Figure 1). From the 72 16S rRNA reads that were generated the majority of reads were from Staphylococcus sp. (56 reads, $78 \%$ of total reads), while the remainder were from Rickettsiella sp. (nine reads, $12.5 \%$ of total reads) and single reads (one read for each) to the following genera: Blautia;
Clostridium; Devosia; Paenalcaligenes; Salinicoccus; Streptococcus; and Tsukamurella (Figure 1). Previous studies of the D. gallinae microbiome have identified Rickettsiella in all life-stages, including eggs, from mites collected from four geographically isolated commercial laying hen facilities in Czechia (Hubert et al., 2017). Rickettsiella is an obligate intracellular bacterium, therefore, it is not likely to be surface associated but found within cells of the mite egg, this raises the possibility that Rickettsiella is maternally inherited in D. gallinae.

\section{Rickettsiella Infection Is Widespread in European Populations of Dermanyssus gallinae}

We performed an extensive diagnostic PCR screen to test D. gallinae from collection sites throughout Europe for the presence of Rickettsiella. To do this, we used a previously produced D. gallinae DNA archive from mites sourced from commercial laying hen facilities from 63 sites across 15 European countries (Karp-Tatham et al., 2020). For each sample site, total D. gallinae DNA from a single adult mite was screened by diagnostic PCR using Rickettsiella-specific 16S rRNA primers. All D. gallinae DNA samples were Rickettsiella positive $(n=63)$, indicating that Rickettsiella infection is widespread in European D. gallinae populations (Figure 2). It is known that other animal- and plant-parasitic arthropods are associated with Rickettsiella. For example, non-pathogenic strains of Rickettsiella have been reported in the pea aphid Acyrthosiphon pisum (Tsuchida et al., 2010, 2014), leafhopper Orosius albicinctus (Iasur-Kruh et al., 2013), and ticks Ixodes woodi and Ixodes uriae (Kurtti et al., 2002; Duron et al., 2016). These strains of Rickettsiella are maternally inherited and can reach high frequencies in natural populations (Kurtti et al., 2002; IasurKruh et al., 2013; Tsuchida et al., 2014; Duron et al., 2016).

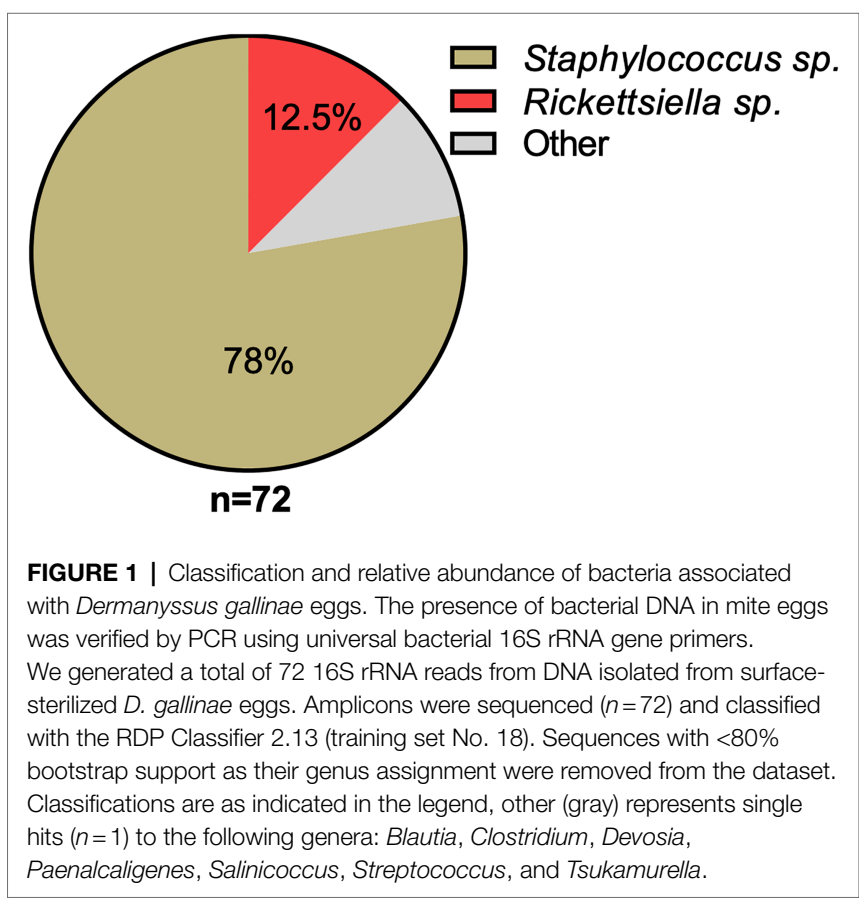


To date, most studies have focused on characterization of Rickettsiella population genetics and association with arthropods through sequence analysis of the $16 \mathrm{~S}$ rRNA gene. Therefore, to gain further insight into the biology of Rickettsiella from D. gallinae, we isolated DNA from mites and completed the Rickettsiella DGE genome sequence.

\section{General Features of the Rickettsiella DGE Genome}

We used previously-generated PacBio long-read sequence data from D. gallinae eggs (Burgess et al., 2018) to assemble the Rickettsiella DGE genome. From a total of $64.0 \mathrm{Gbp}$ of sequence data, $1.3 \mathrm{Gbp}$ of reads did not map to the D. gallinae draft genome and were used for metagenome assembly. The metagenome assembly contained 652 contigs and included a circular Rickettsiella DGE chromosome of $1.89 \mathrm{Mbp}$. To correct errors associated with long-read sequence data, the Rickettsiella DGE assembly was polished using five iterative rounds of Pilon with DNBSEQ ${ }^{\mathrm{TM}}$ short-read sequence data from symbiont enriched DNA. This yielded a circular chromosome of $1,888,715$ bp with $3,712 \times$ coverage and a $\mathrm{G}+\mathrm{C}$ content of $39.6 \%$ (Figure 3). Based on Prokka gene prediction and annotation, the Rickettsiella DGE genome has 1,973 protein coding open reading frames (ORFs) with an average size of $870 \mathrm{bp}$ which covered $91 \%$ of the genome (Table 1; Supplementary Table 1). Of these ORFs, 970 were assigned a biological function by Prokka annotation, 585 were annotated by BLAST homology to characterized proteins, while 227 matched hypothetical proteins of unknown function and 191 were unique

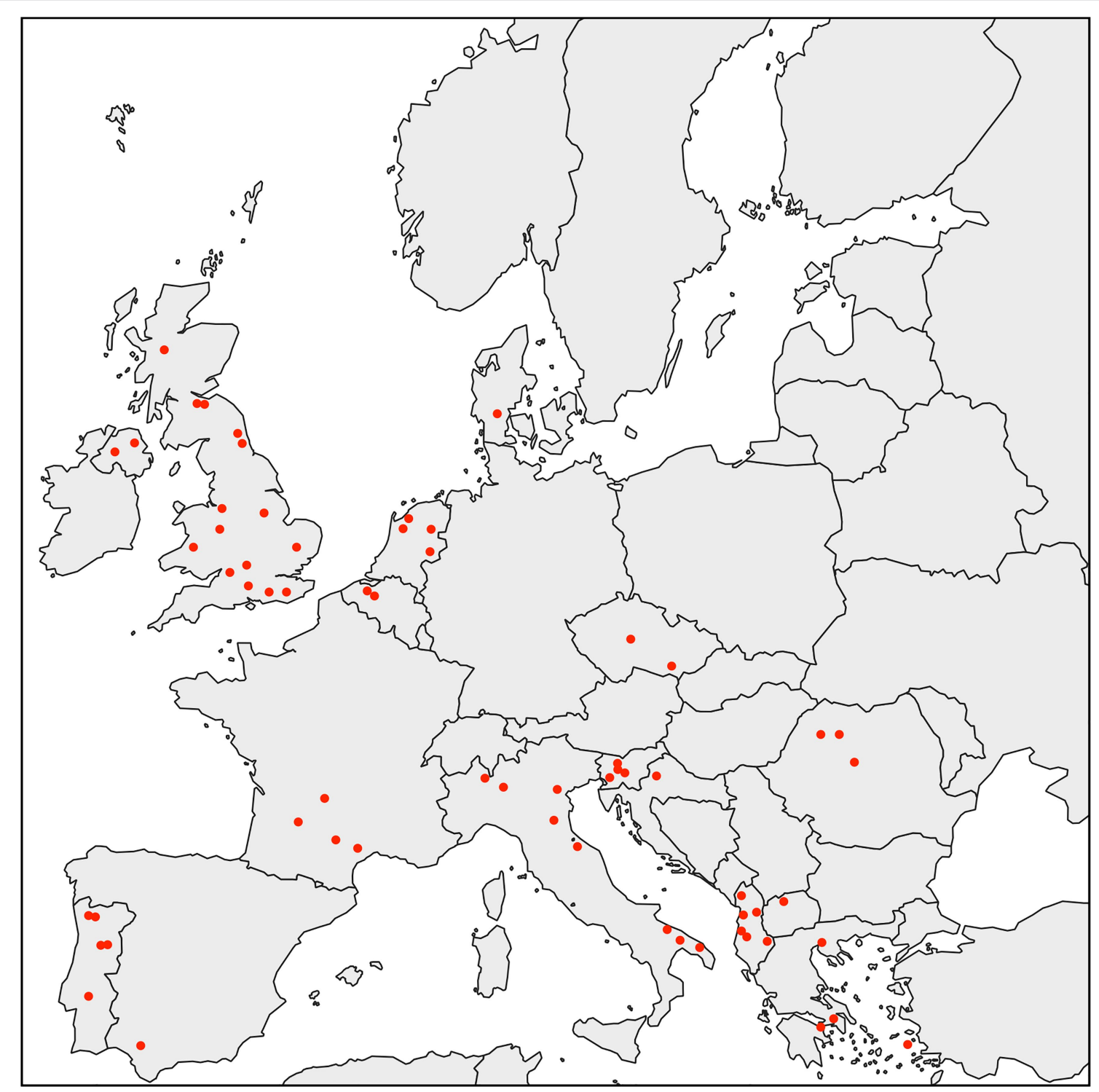

FIGURE 2 | Map showing the distribution of $D$. gallinae populations analyzed in this study. All individual adult female $D$. gallinae mites from each sampling site (63 sites across Europe) were positive for Rickettsiella infection (red circle) suggesting that Rickettsiella infection may have reached fixation in European D. gallinae populations. 
to Rickettsiella DGE. In seven cases, pairs of adjacent genes were annotated with identical names and clearly the ORF was interrupted by a stop codon splitting the gene into two or more parts (these genes are highlighted in Supplementary Table 1). It is likely that these fragmented genes are non-functional and in the early stages of pseudogenization. Other candidate pseudogenes were identified based on their length ratios of each predicted Rickettsiella DGE protein against their top blastp hit from searches against the NCBI nr protein database. In summary, out of a total of 1,973 Rickettsiella DGE protein coding ORFs searched, only 312 (15.8\%) deviate by more than $+/-25 \%$ from their top hit and are candidate pseudogenes (Supplementary Table 1). However, it should be noted that the majority of these pseudogene candidates are "hypothetical proteins" of unknown function and, therefore, await experimental validation as genuine loss of function pseudogenes. We detected 41 tRNA genes (which can translate all 61 amino acid codons), six rRNA gene operons and 19 IS elements. Rickettsiella DGE contains 19 IS elements evenly distributed around the genome and there are eight copies of IS256 family transposase; four IS481; four ISNCY; and three IS5. Of these IS elements, two IS5 family elements have identical nucleotide sequences (OFBDPGAJ_01174 and OFBDPGAJ_01246) and seven IS256 family elements have identical nucleotide sequences (OFBDPGAJ_00304; OFBDPGAJ_00358; OFBDPGAJ_00392; OFBDPGAJ_00512; OFBDPGAJ_01167; OFBDPGAJ_01364; and OFBDPGAJ_01423). Due to high sequence similarity, it is likely that these IS elements are recently active in the Rickettsiella DGE genome.

\section{Rickettsiella DGE Is Related to Endosymbionts and Endoparasites From the Order Legionellales}

Our phylogenetic analysis, using 16S rRNA gene sequences from representative Gammaproteobacteria, supports the placement Rickettsiella DGE within the Rickettsiella genus (Figure 4).

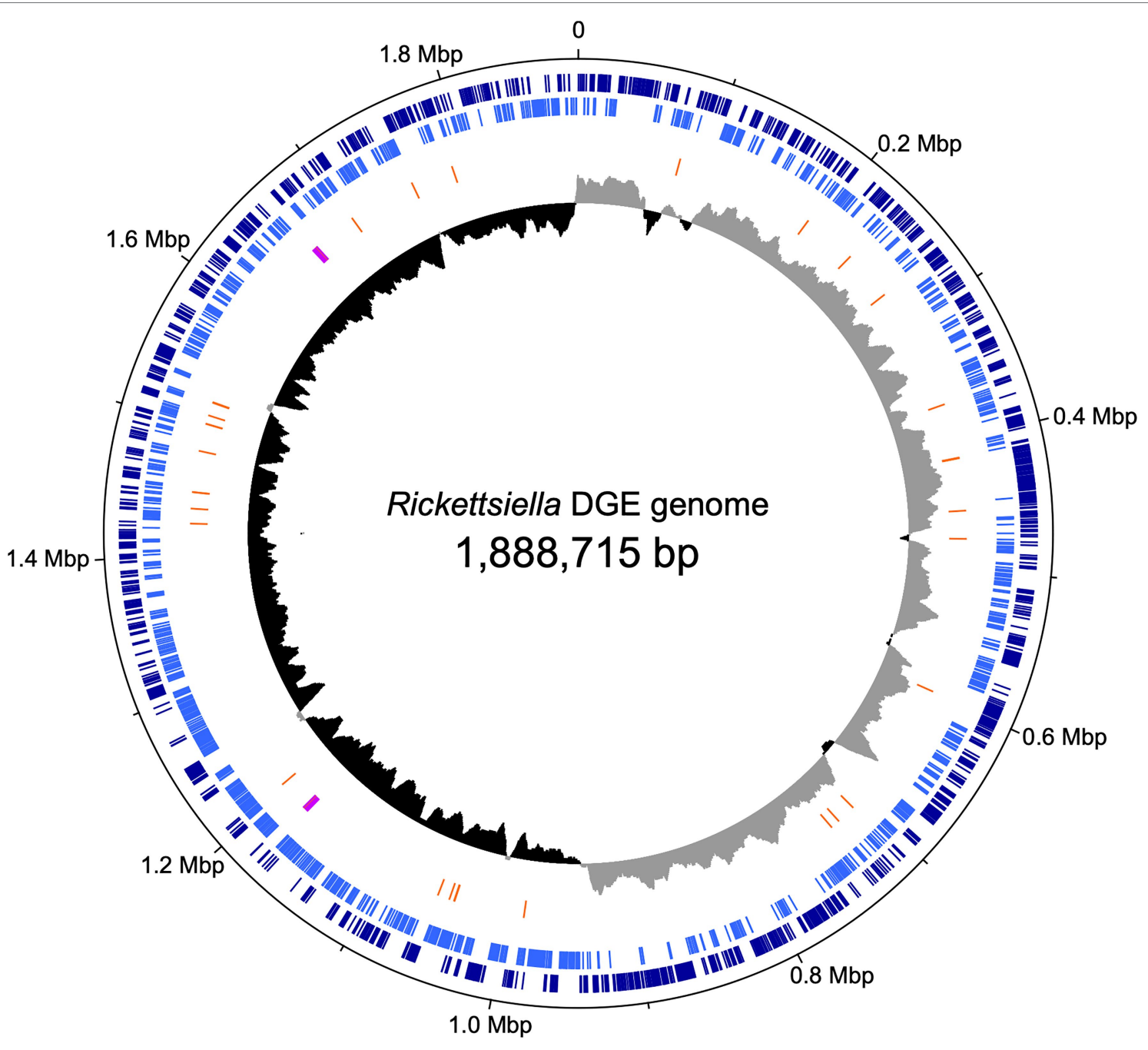

FIGURE 3 | Map of the circular chromosome of Rickettsiella DGE. The innermost circle shows GC skew (window size: 10,000 bp) with gray and black indicating high $(>0)$ and low $(<0)(G-C) /(G+C)$ values. The second circle shows the positions of tRNA genes (orange) and rRNA genes (purple). The outer circles indicate the positions of protein coding genes on the plus strand (dark blue) and minus strand (light blue). 
TABLE 1 | General genomic features of Rickettsiella DGE and allied Gammaproteobacteria.

\begin{tabular}{|c|c|c|c|c|}
\hline & $\begin{array}{c}\text { Rickettsiella } \\
\text { DGE }\end{array}$ & R. viridis & $\begin{array}{c}\text { Coxiella burnetii } \\
\text { RSA } 493\end{array}$ & $\begin{array}{c}\text { Escherichia } \\
\text { coli K-12 }\end{array}$ \\
\hline $\begin{array}{l}\text { Genome size, } \\
\text { Mbp }\end{array}$ & 1.89 & 1.58 & 2.00 & 4.64 \\
\hline $\mathrm{G}+\mathrm{C}(\%)$ & 39.6 & 39.3 & 42.7 & 50.8 \\
\hline $\begin{array}{l}\text { Protein-coding } \\
\text { genes }\end{array}$ & 1,973 & 1,362 & 1,798 & 4,242 \\
\hline $\begin{array}{l}\text { Number of } \\
\text { COGs }^{\#}\end{array}$ & 1,322 & 1,033 & 1,293 & 3,812 \\
\hline $\begin{array}{l}\text { Coding density } \\
(\%)\end{array}$ & 91.0 & 87.1 & 77.7 & 85.8 \\
\hline $\begin{array}{l}\text { Average gene } \\
\text { size }\end{array}$ & 870 & 1,010 & 862 & 939 \\
\hline
\end{tabular}

"COG, cluster of orthologous groups.

Members of the Rickettsiella genus form a monophyletic group that diverged from C. burnetii, the etiologic agent of $\mathrm{Q}$ fever, approximately 350 million years ago (Cordaux et al., 2007). Rickettsiella sp. are found in a wide range of arthropod hosts and are best known as obligate intracellular pathogens (Cordaux et al., 2007; Leclerque and Kleespies, 2008), though, recently, some have been characterized as mutualistic endosymbionts (Tsuchida et al., 2010; Duron et al., 2015). Based on phylogenetic analysis using $16 \mathrm{~S}$ rRNA sequences, Rickettsiella DGE is closely related to Rickettsiella that was isolated from D. gallinae from commercial egg laying facilities in Czechia (Hubert et al., 2017). Furthermore, all Rickettsiella strains from D. gallinae are closely related to Rickettsiella of the tick I. uriae (Duron et al., 2016) and $R$. viridis of the pea aphid, A. pisum (Nikoh et al., 2018; Figure 4). In aphids, $R$. viridis infection is associated with production of blue-green pigment molecules that accumulate in the host (Tsuchida et al., 2010) and not associated with negative impacts on host fitness (Tsuchida et al., 2010). As Rickettsiella present in D. gallinae is closely related to strains found in other species it may indicate horizontal transfer of Rickettsiella across arthropod species (Figure 4). Whole genome alignments reveal shared synteny between Rickettsiella DGE and $R$. viridis, with evidence of genomic rearrangements including inversions, translocations, and insertions (Figure 5).

Rickettsiella DGE is related to other nutritional endosymbionts of blood-feeding arthropods in the order Legionellales. Within the order Legionellales, the CLEs of ticks form a monophyletic group most closely related to the human pathogen $C$. burnetii (Figure 4). In blood-feeding ticks, CLEs are required for the synthesis and supplementation of B vitamins that are lacking in the host's blood meal and are essential for tick survival (Guizzo et al., 2017). In addition, and again within the order Legionellales, the blood-feeding louse Polyplax serrata is associated with a vertically transmitted, host restricted, nutritional endosymbiont from the genus Legionella (Ř́hová et al., 2017; Figure 4). In $P$. serrata, these endosymbionts synthesize and provision $\mathrm{B}$ vitamins to their obligate blood-feeding host (Ř́hová et al., 2017). In summary, endosymbiotic bacteria from the order Legionellales are widely associated with blood feeding arthropods.

\section{Genomic Reduction in Rickettsiella DGE: An Ongoing Process?}

Genome reduction is widespread in maternally-inherited bacterial endosymbionts and is associated with loss of genes that are functionally redundant with the host, resulting in compact endosymbiont genomes containing a subset of genes relative to their free-living ancestor (McCutcheon and Moran, 2012). The genome of Rickettsiella DGE (1.89 Mbp) and $R$. viridis $(1.6 \mathrm{Mbp})$ are both moderately reduced in comparison to C. burnetii (2.03 Mbp; Table 1). Although, it should be noted that $C$. burnetii is already host-adapted as an obligate intracellular parasite and, as such, compared to free-living bacteria it has a degenerate genome (Seshadri et al., 2003). Again, relative to C. burnetii, CLEs of blood-feeding ticks have reduced genomes, retaining functionally non-redundant genes that are essential for the symbiosis. Recent genome sequencing studies unveiled that, in comparison to C. burnetii (genome size $2.03 \mathrm{Mbp}$ ), CLEs from ticks exhibit genome reduction, with genomes ranging from $0.66 \mathrm{Mbp}$ for Coxiella sp. strain CLEAA (CLE of A. americanum; Smith et al., 2015) to $1.73 \mathrm{Mbp}$ for Coxiella sp. strain CRt (CLE of Rhipicephalus turanicus; Gottlieb et al., 2015). Presumably the range of genome size among CLEs of blood-feeding ticks reflects an ongoing dynamic process of reductive genome evolution. Metabolic reconstruction of these reduced genomes reveals intact B vitamin biosynthesis pathways, required for biosynthesis and provision of these essential nutrients to the host tick (Gottlieb et al., 2015; Smith et al., 2015).

Perhaps the most striking example of genome reduction, in the transition from a pathogen to a nutritional mutualist, is the loss of virulence associated secretion systems. In the pathogens C. burnetii and Legionella pneumophila the type IV Dot/Icm secretion system (T4SS) functions to export a suite of virulence factors that modulate host physiology and are essential for establishment and maintenance of infection (Seshadri et al., 2003; Chien et al., 2004; Gomez-Valero et al., 2019). Intriguingly, the massively reduced genomes of Coxiella from the lone star tick A. americanum (CLEAA) and Legionella polyplacis from the bloodfeeding louse $P$. serrata do not encode a Dot/Icm type IVB secretion system and presumably this secretion apparatus is not required in these nutritional mutualists (Smith et al., 2015; Ríhová et al., 2017). In contrast, components of the Dot/Icm type IVB secretion system are retained in Rickettsiella DGE and are also present in the closely related genome of $R$. viridis, although, the sequences of core components are highly divergent when compared with L. pneumophila orthologs (Supplementary Table 2). It therefore remains to be determined if the Dot/Icm type IVB secretion system is functional in Rickettsiella DGE and the role it plays in cellular interactions with the host.

\section{Metabolic Capacity of Rickettsiella DGE: A Putative Nutritional Mutualist}

The Rickettsiella DGE genome, as with the related intracellular facultative symbiont $R$. viridis, retains genes for basic cellular processes including translation, replication, cell wall biosynthesis, and energy production (Figure 6). In Supplementary Table 3, we provide a more detailed comparative gene content analysis 


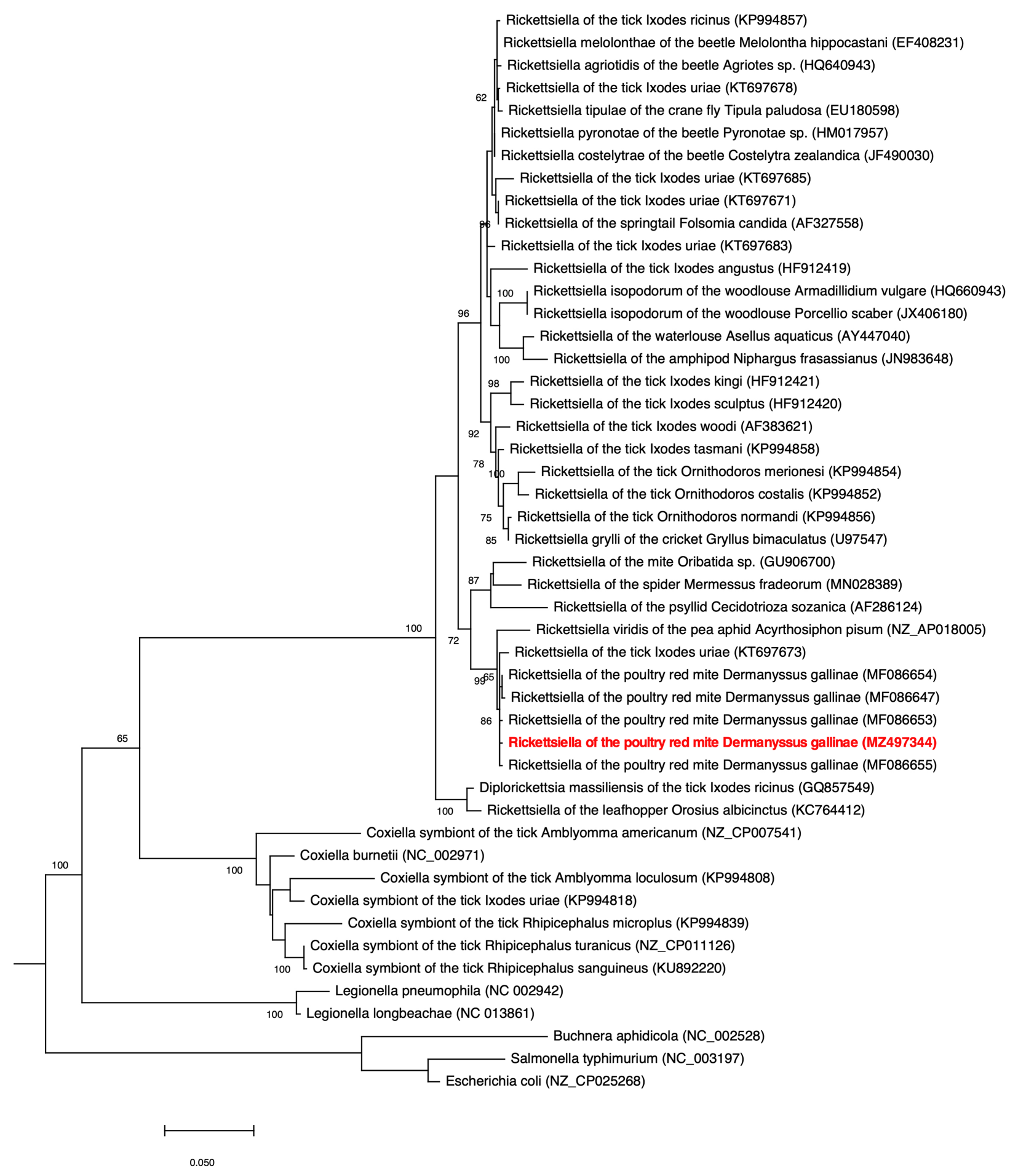

Rickettsiella of the tick Ixodes ricinus (KP994857)

Rickettsiella melolonthae of the beetle Melolontha hippocastani (EF408231)

Rickettsiella agriotidis of the beetle Agriotes sp. (HQ640943)

Rickettsiella tipulae of the crane fly Tipula paludosa (EU180598)

- Rickettsiella of the tick Ixodes uriae (KT697685)

(AF327558)

kettsiella of the tick lxodes uriae (KT697683)

Rickettsiella of the tick Ixodes angustus (HF912419)

Rickettsiella isopodorum of the woodlouse Porcellio scaber (JX406180)

100 Rickettsiella of the amphipod Niphargus frasassianus (JN983648)

Rickettsiella of the tick Ornithodoros normandi (KP994856)

85 Rickettsiella grylli of the cricket Gryllus bimaculatus (U97547)

(GU906700) - Rickettsiella of the psyllid Cecidotrioza sozanica (AF286124)

Diplorickettsia massiliensis of the tick Ixodes ricinus (GQ857549)

FIGURE 4 | Phylogenetic placement of Rickettsiella DGE in the Gammaproteobacteria. The maximum likelihood phylogeny is inferred from 16S rDNA sequences (1,013 unambiguously aligned nucleotide sites). Statistical support is shown at each node from 1,000 bootstrap replicates (bootstrap values $>60 \%$ are shown). The Rickettsiella DGE sequence highlighted in red (MZ497344) was generated in the current study. Accession numbers are indicated in brackets. Scale bar represents 0.02 substitutions per site.

between Rickettsiella DGE and genomes of $R$. viridis and C. burnetii using the pathway/gene list published by Moran et al. (2008) and Bennett and Moran (2013).
Metabolic reconstruction of amino acid biosynthesis pathways revealed that Rickettsiella DGE is unable to synthesize protein amino acids and, therefore, amino acids are likely provisioned 


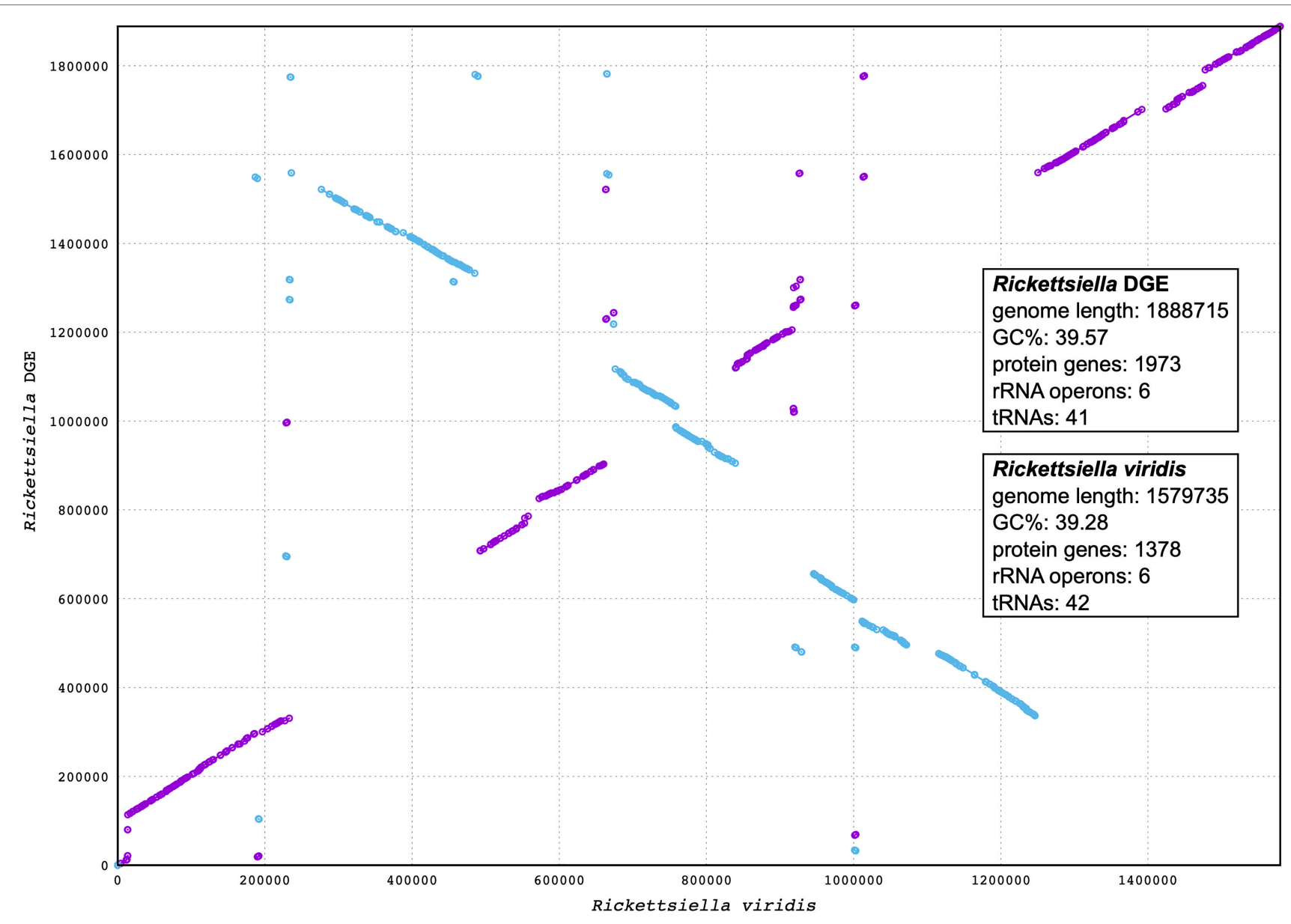

FIGURE 5 | Synteny analysis between Rickettsiella DGE and Rickettsiella viridis genomes. The Rickettsiella DGE genome is represented on the $y$ axis and the $R$. viridis genome is represented on the $x$ axis. Blue and purple lines represent synteny between the two genomes, with blue lines being inverted in Rickettsiella DGE relative to $R$. viridis.

by the host (Figure 7). The biosynthesis pathway for the essential amino acid lysine is mostly complete (8/9 required genes present), although, precursor aspartic acid in not synthesized by Rickettsiella DGE and the bifunctional aspartokinase/homoserine dehydrogenase 1 (encoded by thrA) is missing, again suggesting this pathway is non-functional. Given that D. gallinae feeds on blood and is able to digest hemoglobin and other blood proteins to release free amino acids (Price et al., 2019), it likely has an excess of essential and non-essential amino acids that meet its own nitrogen requirements and those of Rickettsiella DGE. Indeed, in other nutritional endosymbionts of obligate blood feeding arthropods, amino acid biosynthesis pathways are absent and it is likely the host supplies amino acids to the endosymbiont (Chien et al., 2004; Smith et al., 2015; Duron et al., 2018).

Obligate blood feeding arthropods such as the human body louse (Pediculus humanus; Kirkness et al., 2010), African soft tick (O. moubata; Duron et al., 2018) and the Lone star tick (A. americanum; Smith et al., 2015) depend on nutritional endosymbionts to synthesize and provide $B$ vitamins that are available in trace amounts in mammalian blood (reviewed in
Husnik, 2018). Thus, to determine whether Rickettsiella DGE is able to synthesize B vitamins, we surveyed its genome for $\mathrm{B}$ vitamin biosynthesis genes. The Rickettsiella DGE genome has conserved genes involved in the biosynthesis of seven B vitamins, including complete biosynthetic pathways for thiamine (vitamin B1) via the salvage pathway, riboflavin (vitamin B2), pyridoxine (vitamin B6) and the cofactors FAD, and CoA (Figure 7). The biosynthesis pathway for biotin (vitamin B7) is largely complete (9/10 genes present), although, it is missing bioH, which is required for pimeloyl-CoA synthesis. The annotated biotin biosynthesis pathway is based on that of the model organism E. coli, where bioC and bioH are required for synthesis of the intermediate pimeloyl-CoA. However, unlike the representative "bioC/bioH" pathway of $\mathrm{E}$. coli many bioCcontaining microorganisms lack bioH homologs, raising the possibility of non-homologous gene replacement in some bacteria (Shapiro et al., 2012). To date, there are five documented cases of bioH gene replacement, which includes bioK of Synechococcus (Shapiro et al., 2012), bioG of Haemophilus influenzae (Shapiro et al., 2012), bioJ of Francisella sp. (Feng et al., 2014), bioV of Helicobacter sp. (Bi et al., 2016), and bioZ of Agrobacterium 


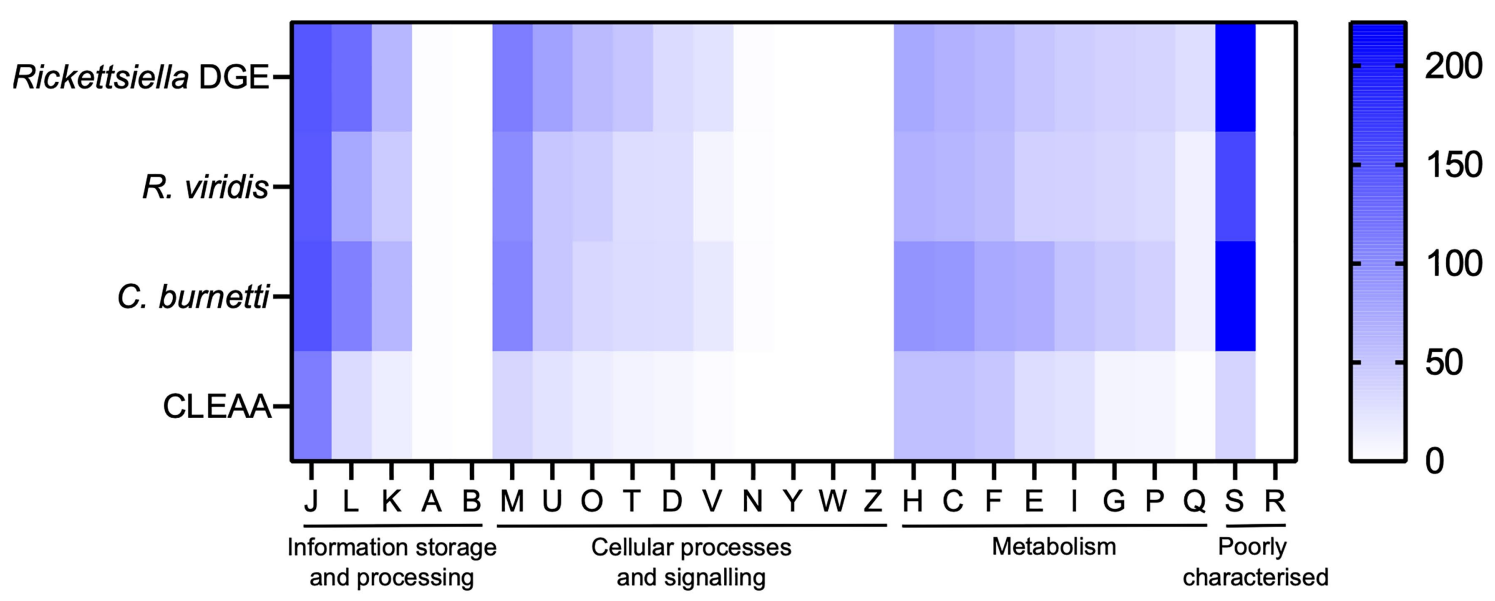

FIGURE 6 | Heatmap comparison of cluster of orthologous groups (COG) frequency in Rickettsiella DGE and related bacteria. Abbreviations for functional categories are as follows: J, translation, ribosomal structure, and biogenesis; L, replication, recombination, and repair; K, transcription; A, RNA processing and modification; B, chromatin structure and dynamics; $\mathrm{M}$, cell wall/membrane/envelope biogenesis; $\mathrm{U}$, intracellular trafficking, secretion, and vesicular transport; $\mathrm{T}$, signal transduction mechanisms; O, posttranslational modification, protein turnover, chaperones; $\mathrm{D}$, cell cycle control, cell division, chromosome partitioning; $\mathrm{V}$, defense mechanisms; N, cell motility; Y, nuclear structure; W, extracellular structures; Z, cytoskeleton; $\mathrm{H}$, coenzyme transport and metabolism; C, energy production and conversion; F, nucleotide transport and metabolism; E, amino acid transport and metabolism; I, lipid transport and metabolism; G, carbohydrate transport and metabolism; P, inorganic ion transport and metabolism; Q, secondary metabolites biosynthesis, transport, and catabolism; S, function unknown; and R, general function prediction only. Scale bar (0, white; 200 , blue) indicates number of COGs in each category.

tumefaciens (Hu and Cronan, 2020). Further tblastn searches against the Rickettsiella DGE genome using bioH and the non-homologous gene replacements bioK, bioG, bioJ, and bioV did not identify gene products that can fill the bioH gap. However, a gene encoding ketoacyl-ACP synthase (KAS) III from Rickettsiella DGE (gene locus OFBDPGAJ_01014) has similarity to bioZ of $A$. tumefaciens ( $53.8 \%$ amino acid similarity) and is therefore a candidate to replace bioH. Alignments between A. tumefaciens KAS III (bioZ) and orthologs from Rickettsiella DGE as well as other Rickettsiella sp. are shown in Supplementary Figure 1. Given the retention of a long biotin biosynthesis pathway in Rickettsiella DGE (9/10 genes present) and the propensity for the missing bioH gene to be replaced in other bacteria, we predict that the biotin biosynthesis pathway is functional in Rickettsiella DGE. In contrast, the other B vitamin biosynthesis pathways for nicotinic acid (vitamin B3), pantothenic acid (vitamin B5), and folic acid (vitamin B9) are more fragmented and it is not clear if these pathways are functional.

In other nutritional host/endosymbiont interactions it has been shown that some fragmented metabolic pathways of nutritional endosymbionts are functional with gene products supplemented from multiple species including the host and/ or symbiont partners. This complex arrangement results in metabolic mosaics for the synthesis of essential nutrients (McCutcheon et al., 2009; Husnik et al., 2013). By utilizing the D. gallinae genome (Burgess et al., 2018), we investigated whether host gene products are capable of filling missing steps in Rickettsiella DGE B vitamin biosynthesis pathways. In general, animals cannot synthesize B vitamins de novo, therefore, we explored the possibility that D. gallinae has acquired genes through horizontal gene transfer (HGT) that allows these fragmented pathways to function. To screen for potential HGT events, we used E. coli proteins from each of the missing steps in Rickettsiella DGE B vitamin biosynthesis as "query" proteins in blastp searches against predicted proteins from the D. gallinae genome (Burgess et al., 2018). These searches did not identify candidate genes from D. gallinae and it is therefore unlikely that D. gallinae contributes to $\mathrm{B}$ vitamin biosynthesis by completing these missing steps. Another possibility is that fragmented B vitamin pathways in Rickettsiella DGE are completed by gene products from other endosymbionts of the mite. A previous microbiome analysis of $D$. gallinae identified several additional endosymbionts (including Bartonella, Cardinium, and Wolbachia) that are prevalent in mite populations (Hubert et al., 2017). However, the biosynthetic capability of these D. gallinae endosymbionts is currently unknown (Hubert et al., 2017). Thus, future work will analyze B vitamin biosynthesis in the context of the D. gallinae metagenome.

In addition to blood feeding ticks and mites, many insects are specialist blood feeders (reviewed in Husnik, 2018). To utilize their blood diet, obligate blood feeding insects also associate with mutualistic endosymbionts that are important for provision of B vitamins to the host (Akman et al., 2002; Kirkness et al., 2010; Nikoh et al., 2014). While endosymbiotic partners differ, there are many commonalities between endosymbiotic partners from blood-feeding ticks, mites, and insects (Husnik, 2018). For example, the tsetse fly (Glossina morsitans) is critically reliant on its obligate endosymbiont Wigglesworthia glossinidia. Elimination of the symbiont using antibiotic treatment results in reproductive failure of the tsetse host (Rio et al., 2016). Critically, it has been shown that reproduction can by partially restored in these flies by dietary supplementation with B vitamins, suggesting that the 
A

Essential amino acids

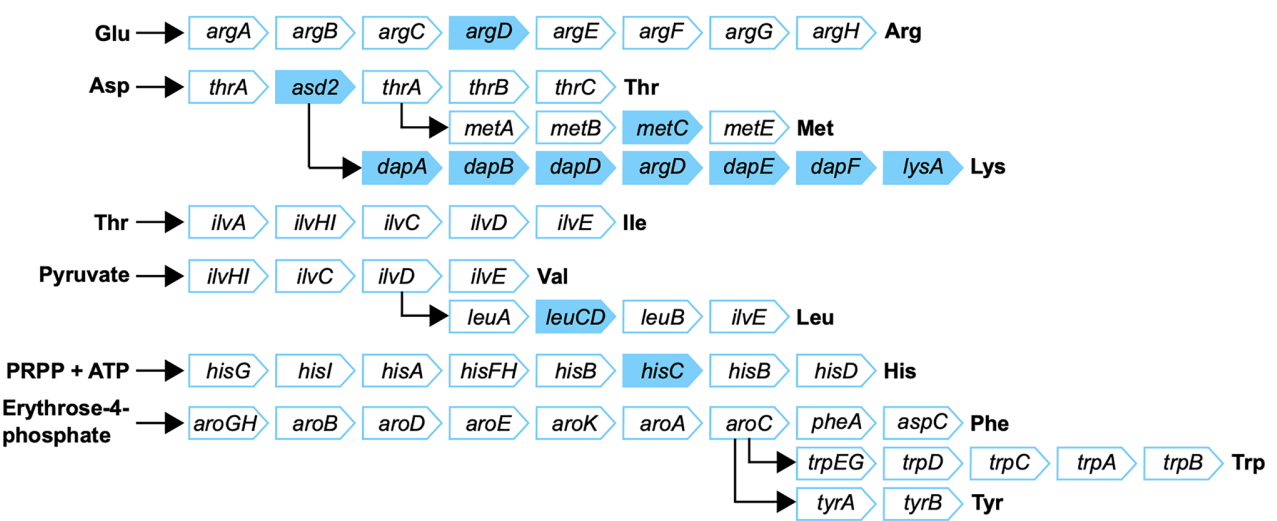

B

Non-essential amino acids
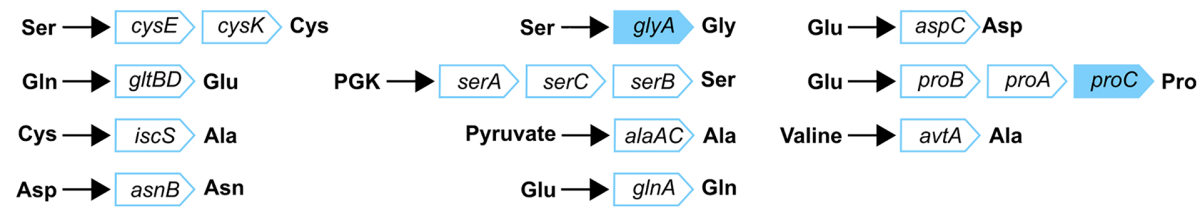

C

Vitamins

AiR $\rightarrow$ thiC thiD thiE thil B1 (Thiamine)

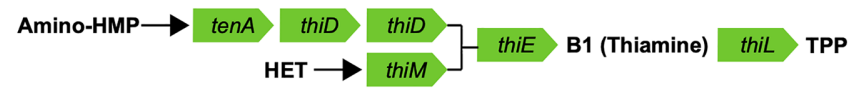

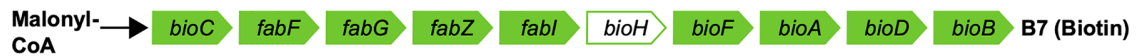

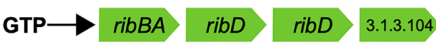

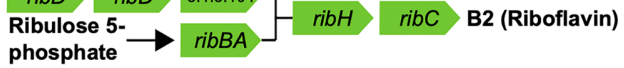

Glutamine $\rightarrow p d x T \quad p d x S \quad$ PLP $\quad p d x Y$ PL

Aspartate $\longrightarrow \operatorname{nadB} \operatorname{nadA} \operatorname{nadC} \operatorname{pncC}$ B3 (Nicotinic acid)

Valine $\longrightarrow$ ilvE panB panE panC B5 (Pantothenic acid)

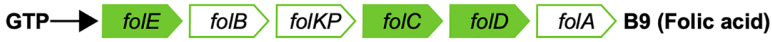

D

\section{Cofactors}

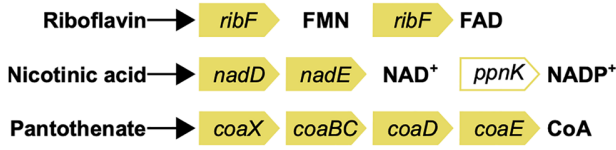

FIGURE 7 | Biosynthetic pathways for synthesis of (A) essential amino acids; (B) non-essential amino acids; (C) vitamins; and (D) cofactors in Rickettsiella DGE. Gene names are indicated in arrowed rectangles; colored arrows show genes present in Rickettsiella DGE; missing genes are shown in white arrows.

endosymbiont may provision these nutrients (Rio et al., 2016). Furthermore, in support of its role as a nutritional mutualist, the genome of Wigglesworthia reveals that the small $700 \mathrm{Kbp}$ endosymbiont genome retains the capability to synthesise B vitamins (Akman et al., 2002; Rio et al., 2012). In comparison to Rickettsiella DGE, where we observe several incomplete B vitamin biosynthesis pathways (Figure 7), Wigglesworthia has complete pathways for the synthesis of biotin (vitamin B7), thiamine (vitamin B1), riboflavin (vitamin B2), pantothenic acid (vitamin B5), and pyridoxine (vitamin B6; Akman et al., 2002;
Rio et al., 2012). Although speculative, this may reflect differing requirements of the host for $\mathrm{B}$ vitamin supplementation across these host/endosymbiont systems. Indeed, genome analysis of endosymbionts from other blood feeding arthropods reveals differing levels of completeness of retained B vitamin biosynthesis pathways (Kirkness et al., 2010; Nikoh et al., 2014; Smith et al., 2015; Duron et al., 2018). A recent analysis of endosymbiont genomes from obligate blood feeding arthropods reveals that all genomes analyzed retain "core" biosynthesis pathways for biotin (vitamin B7) and to a lesser degree folic acid (vitamin B9) 
and riboflavin (vitamin B2; Duron and Gottlieb, 2020). In the analysis by Duron and Gottlieb (2020), other B vitamin pathways were more fragmented and pathway functionality may reflect the lifestyle of the host and its nutritional requirement for $\mathrm{B}$ vitamin supplementation. In both Rickettsiella DGE and other endosymbionts from blood feeding arthropods further investigation is needed to understand if and how these fragmented $B$ vitamin biosynthetic pathways are functional as well as the exact $\mathrm{B}$ vitamin requirements of each host.

Currently, in the D. gallinae - Rickettsiella DGE endosymbiotic system the tissue location of Rickettsiella DGE is unknown, as is the identity of host genes required for the maintenance of the association. Again, this is something that has been extensively investigated in the tsetse/Wigglesworthia interaction (Bing et al., 2017). In teste flies, Wigglesworthia is located in host bacteriocyte cells that collectively form the bacteriome organ in the anterior midgut (Rio et al., 2012). Dual analysis of the host/endosymbiont transcriptome identified host factors that contribute to the maintenance of the symbiosis and a multivitamin transporter potentially involved in nutrient provision to the host (Bing et al., 2017). In support of its role as a nutritional mutualist, genes involved in biosynthesis of $\mathrm{B}$ vitamins and co-factors were highly expressed by the endosymbiont (Bing et al., 2017). Thus, the key priorities for future research are to determine the molecular processes underpinning maintenance of Rickettsiella DGE in host cells and the genetic and metabolic mechanisms by which nutrient flux between host and endosymbiont is regulated.

\section{DATA AVAILABILITY STATEMENT}

DNBseq reads were deposited to the Sequence Read Archive (SRA), under NCBI BioProject PRJNA743410. The genome sequence of Rickettsiella DGE has been deposited at GenBank with the

\section{REFERENCES}

Akman, L., Yamashita, A., Watanabe, H., Oshima, K., Shiba, T., Hattori, M., et al. (2002). Genome sequence of the endocellular obligate symbiont of tsetse flies, Wigglesworthia glossinidia. Nat. Genet. 32, 402-407. doi: 10.1038/ng986

Bennett, G. M., and Moran, N. A. (2013). Small, smaller, smallest: the origins and evolution of ancient dual symbioses in a phloem-feeding insect. Genome Biol. Evol. 5, 1675-1688. doi: 10.1093/gbe/evt118

Bi, H., Zhu, L., Jia, J., and Cronan, J. E. (2016). A biotin biosynthesis gene restricted to helicobacter. Sci. Rep. 6:21162. doi: 10.1038/srep21162

Bing, X., Attardo, G. M., Vigneron, A., Aksoy, E., Scolari, F., Malacrida, A., et al. (2017). Unravelling the relationship between the tsetse fly and its obligate symbiont Wigglesworthia: transcriptomic and metabolomic landscapes reveal highly integrated physiological networks. Proc. Biol. Sci. 284:20170360. doi: $10.1098 / \mathrm{rspb} .2017 .0360$

Burgess, S. T. G., Bartley, K., Nunn, F., Wright, H. W., Hughes, M., Gemmell, M., et al. (2018). Draft genome assembly of the poultry red mite, Dermanyssus gallinae. Microbiol. Resour. Announc. 7:e01221-18. doi: 10.1128/MRA.01221-18

Carver, T., Thomson, N., Bleasby, A., Berriman, M., and Parkhill, J. (2009). DNAPlotter: circular and linear interactive genome visualization. Bioinformatics 25, 119-120. doi: 10.1093/bioinformatics/btn578

Caspi, R., Foerster, H., Fulcher, C. A., Hopkinson, R., Ingraham, J., Kaipa, P., et al. (2006). MetaCyc: a multiorganism database of metabolic pathways and enzymes. Nucleic Acids Res. 34, D511-D516. doi: 10.1093/nar/gkj128 accession number CP079094. Rickettsiella DGE 16S rRNA generated in this study is available from GenBank under the following accession numbers: MZ497336-MZ497344.

\section{AUTHOR CONTRIBUTIONS}

DP, AN, and SB conceived the study and analyzed the data. DP, $\mathrm{KB}, \mathrm{DB}, \mathrm{EK}-\mathrm{T}, \mathrm{FN}, \mathrm{SB}$, and AN designed the research. DP and EK-T performed the research. DP wrote the paper with contributions from all authors. All authors contributed to the article and approved the submitted version.

\section{FUNDING}

The work was supported by a Moredun Foundation Research fellowship awarded to DP and a British Egg Marketing Board (BEMB) Trust PhD scholarship awarded to EK-T.

\section{ACKNOWLEDGMENTS}

We thank the Bioservices Group at Moredun Research Institute for their ongoing help and expertise and United Kingdom farmers for allowing access to sites for D. gallinae collection. We thank Alex Wilson (University of Miami) for helpful discussion during the course of this project.

\section{SUPPLEMENTARY MATERIAL}

The Supplementary Material for this article can be found online at: https://www.frontiersin.org/articles/10.3389/fmicb.2021.695346/ full\#supplementary-material

Chien, M., Morozova, I., Shi, S., Sheng, H., Chen, J., Gomez, S. M., et al. (2004). The genomic sequence of the accidental pathogen Legionella pneumophila. Science 305, 1966-1968. doi: 10.1126/science.1099776

Cordaux, R., Paces-Fessy, M., Raimond, M., Michel-Salzat, A., Zimmer, M., and Bouchon, D. (2007). Molecular characterization and evolution of arthropod-pathogenic rickettsiella bacteria. Appl. Environ. Microbiol. 73, 5045-5047. doi: 10.1128/AEM.00378-07

De Luna, C. J., Moro, C. V., Guy, J. H., Zenner, L., and Sparagano, O. A. E. (2009). Endosymbiotic bacteria living inside the poultry red mite (Dermanyssus gallinae). Exp. Appl. Acarol. 48, 105-113. doi: 10.1007/s10493-008-9230-2

Delcher, A. L., Phillippy, A., Carlton, J., and Salzberg, S. L. (2002). Fast algorithms for large-scale genome alignment and comparison. Nucleic Acids Res. 30, 2478-2483. doi: 10.1093/nar/30.11.2478

Duron, O., Cremaschi, J., and McCoy, K. D. (2016). The high diversity and global distribution of the intracellular bacterium Rickettsiella in the polar seabird tick Ixodes uriae. Microb. Ecol. 71, 761-770. doi: 10.1007/s00248-015-0702-8

Duron, O., and Gottlieb, Y. (2020). Convergence of nutritional symbioses in obligate blood feeders. Trends Parasitol. 36, 816-825. doi: 10.1016/j. pt.2020.07.007

Duron, O., Morel, O., Noël, V., Buysse, M., Binetruy, F., Lancelot, R., et al. (2018). Tick-bacteria mutualism depends on B vitamin synthesis pathways. Curr. Biol. 28, 1896.e5-1902.e5. doi: 10.1016/j.cub.2018.04.038

Duron, O., Noël, V., McCoy, K. D., Bonazzi, M., Sidi-Boumedine, K., Morel, O., et al. (2015). The recent evolution of a maternally-inherited endosymbiont 
of ticks led to the emergence of the Q fever pathogen, Coxiella burnetii. PLoS Pathog. 11:e1004892. doi: 10.1371/journal.ppat.1004892

Feng, Y., Napier, B. A., Manandhar, M., Henke, S. K., Weiss, D. S., and Cronan, J. E. (2014). A Francisella virulence factor catalyses an essential reaction of biotin synthesis. Mol. Microbiol. 91, 300-314. doi: 10.1111/mmi.12460

George, D. R., Finn, R. D., Graham, K. M., Mul, M. F., Maurer, V., Moro, C. V., et al. (2015). Should the poultry red mite Dermanyssus gallinae be of wider concern for veterinary and medical science? Parasit. Vectors 8:178. doi: 10.1186/s13071-015-0768-7

Gomez-Valero, L., Rusniok, C., Carson, D., Mondino, S., Pérez-Cobas, A. E., Rolando, M., et al. (2019). More than 18,000 effectors in the Legionella genus genome provide multiple, independent combinations for replication in human cells. Proc. Natl. Acad. Sci. U. S. A. 116, 2265-2273. doi: 10.1073/ pnas. 1808016116

Gottlieb, Y., Lalzar, I., and Klasson, L. (2015). Distinctive genome reduction rates revealed by genomic analyses of two Coxiella-like endosymbionts in ticks. Genome Biol. Evol. 7, 1779-1796. doi: 10.1093/gbe/evv108

Guizzo, M. G., Parizi, L. F., Nunes, R. D., Schama, R., Albano, R. M., Tirloni, L., et al. (2017). A Coxiella mutualist symbiont is essential to the development of Rhipicephalus microplus. Sci. Rep. 7:17554. doi: 10.1038/s41598-017-17309-x

$\mathrm{Hu}, \mathrm{Y}$., and Cronan, J. E. (2020). $\alpha$-Proteobacteria synthesize biotin precursor pimeloyl-ACP using BioZ 3-ketoacyl-ACP synthase and lysine catabolism. Nat. Commun. 11:5598. doi: 10.1038/s41467-020-19251-5

Hubert, J., Erban, T., Kopecky, J., Sopko, B., Nesvorna, M., Lichovnikova, M., et al. (2017). Comparison of microbiomes between red poultry mite populations (Dermanyssus gallinae): predominance of Bartonella-like bacteria. Microb. Ecol. 74, 947-960. doi: 10.1007/s00248-017-0993-Z

Huong, C. T. T., Murano, T., Uno, Y., Usui, T., and Yamaguchi, T. (2014). Molecular detection of avian pathogens in poultry red mite (Dermanyssus gallinae) collected in chicken farms. J. Vet. Med. Sci. 76, 1583-1587. doi: 10.1292/jvms.14-0253

Husnik, F. (2018). Host-symbiont-pathogen interactions in blood-feeding parasites: nutrition, immune cross-talk and gene exchange. Parasitology 145, 1294-1303. doi: $10.1017 / S 0031182018000574$

Husnik, F., Nikoh, N., Koga, R., Ross, L., Duncan, R. P., Fujie, M., et al. (2013). Horizontal gene transfer from diverse bacteria to an insect genome enables a tripartite nested mealybug symbiosis. Cell 153, 1567-1578. doi: 10.1016/j.cell.2013.05.040

Hyatt, D., Chen, G.-L., LoCascio, P. F., Land, M. L., Larimer, F. W., and Hauser, L. J. (2010). Prodigal: prokaryotic gene recognition and translation initiation site identification. BMC Bioinformatics 11:119. doi: 10.1186/14712105-11-119

Iasur-Kruh, L., Weintraub, P. G., Mozes-Daube, N., Robinson, W. E., Perlman, S. J., and Zchori-Fein, E. (2013). Novel Rickettsiella bacterium in the leafhopper Orosius albicinctus (Hemiptera: Cicadellidae). Appl. Environ. Microbiol. 79, 4246-4252. doi: 10.1128/AEM.00721-13

Jaziri, F., Parisot, N., Abid, A., Denonfoux, J., Ribière, C., Gasc, C., et al. (2014). PhylOPDb: a 16S rRNA oligonucleotide probe database for prokaryotic identification. Database 2014:bau036. doi: 10.1093/database/bau036

Kanehisa, M., and Goto, S. (2000). KEGG: kyoto encyclopedia of genes and genomes. Nucleic Acids Res. 28, 27-30. doi: 10.1093/nar/28.1.27

Karp-Tatham, E., Küster, T., Angelou, A., Papadopoulos, E., Nisbet, A. J., Xia, D., et al. (2020). Phylogenetic inference using cytochrome $c$ oxidase subunit I (COI) in the poultry red mite, Dermanyssus gallinae in the United Kingdom relative to a European framework. Front. Vet. Sci. 7:553. doi: 10.3389/ fvets. 2020.00553

Kirkness, E. F., Haas, B. J., Sun, W., Braig, H. R., Perotti, M. A., Clark, J. M., et al. (2010). Genome sequences of the human body louse and its primary endosymbiont provide insights into the permanent parasitic lifestyle. Proc. Natl. Acad. Sci. U. S. A. 107, 12168-12173. doi: 10.1073/pnas.1003379107

Kolmogorov, M., Bickhart, D. M., Behsaz, B., Gurevich, A., Rayko, M., Shin, S. B., et al. (2020). metaFlye: scalable long-read metagenome assembly using repeat graphs. Nat. Methods 17, 1103-1110. doi: 10.1038/s41592-020-00971-x

Kumar, S., Stecher, G., Li, M., Knyaz, C., and Tamura, K. (2018). MEGA X: molecular evolutionary genetics analysis across computing platforms. Mol. Biol. Evol. 35, 1547-1549. doi: 10.1093/molbev/msy096

Kurtti, T. J., Palmer, A. T., and Oliver, J. H. (2002). Rickettsiella-like bacteria in Ixodes woodi (Acari: Ixodidae). J. Med. Entomol. 39, 534-540. doi: 10.1603/0022-2585-39.3.534
Laslett, D., and Canback, B. (2004). ARAGORN, a program to detect tRNA genes and tmRNA genes in nucleotide sequences. Nucleic Acids Res. 32, 11-16. doi: 10.1093/nar/gkh152

Leclerque, A., and Kleespies, R. G. (2008). Type IV secretion system components as phylogenetic markers of entomopathogenic bacteria of the genus Rickettsiella. FEMS Microbiol. Lett. 279, 167-173. doi: 10.1111/j.1574-6968.2007.01025.x

Li, H. (2013). Aligning sequence reads, clone sequences and assembly contigs with BWA-MEM. Available at: http://arxiv.org/abs/1303.3997 (Accessed February 12, 2021).

Li, H. (2018). Minimap2: pairwise alignment for nucleotide sequences. Bioinformatics 34, 3094-3100. doi: 10.1093/bioinformatics/bty191

Li, H., Handsaker, B., Wysoker, A., Fennell, T., Ruan, J., Homer, N., et al. (2009). The sequence alignment/map format and SAMtools. Bioinformatics 25, 2078-2079. doi: 10.1093/bioinformatics/btp352

McCutcheon, J. P., McDonald, B. R., and Moran, N. A. (2009). Convergent evolution of metabolic roles in bacterial co-symbionts of insects. Proc. Natl. Acad. Sci. U. S. A. 106, 15394-15399. doi: 10.1073/pnas.0906424106

McCutcheon, J. P., and Moran, N. A. (2012). Extreme genome reduction in symbiotic bacteria. Nat. Rev. Microbiol. 10, 13-26. doi: 10.1038/ nrmicro2670

McFall-Ngai, M., Hadfield, M. G., Bosch, T. C. G., Carey, H. V., Domazet-Lošo, T., Douglas, A. E., et al. (2013). Animals in a bacterial world, a new imperative for the life sciences. Proc. Natl. Acad. Sci. U. S. A. 110, 3229-3236. doi: 10.1073/pnas.1218525110

Moran, N. A. (2007). Symbiosis as an adaptive process and source of phenotypic complexity. Proc. Natl. Acad. Sci. U. S. A. 104, 8627-8633. doi: 10.1073/ pnas.0611659104

Moran, N. A., McCutcheon, J. P., and Nakabachi, A. (2008). Genomics and evolution of heritable bacterial symbionts. Annu. Rev. Genet. 42, 165-190. doi: 10.1146/annurev.genet.41.110306.130119

Nikoh, N., Hosokawa, T., Moriyama, M., Oshima, K., Hattori, M., and Fukatsu, T. (2014). Evolutionary origin of insect-Wolbachia nutritional mutualism. Proc. Natl. Acad. Sci. U. S. A. 111, 10257-10262. doi: 10.1073/ pnas. 1409284111

Nikoh, N., Tsuchida, T., Maeda, T., Yamaguchi, K., Shigenobu, S., Koga, R., et al. (2018). Genomic insight into symbiosis-induced insect color change by a facultative bacterial endosymbiont, "Candidatus Rickettsiella viridis". mBio 9:e00890-18. doi: 10.1128/mBio.00890-18

Price, D. R. G., Küster, T., Øines, Ø., Oliver, E. M., Bartley, K., Nunn, F., et al. (2019). Evaluation of vaccine delivery systems for inducing long-lived antibody responses to Dermanyssus gallinae antigen in laying hens. Avian Pathol. 48, S60-S74. doi: 10.1080/03079457.2019.1612514

Ř́ihová, J., Nováková, E., Husník, F., and Hypša, V. (2017). Legionella becoming a mutualist: adaptive processes shaping the genome of symbiont in the louse Polyplax serrata. Genome Biol. Evol. 9, 2946-2957. doi: 10.1093/gbe/ evx217

Rio, R. V. M., Attardo, G. M., and Weiss, B. L. (2016). Grandeur alliances: symbiont metabolic integration and obligate arthropod hematophagy. Trends Parasitol. 32, 739-749. doi: 10.1016/j.pt.2016.05.002

Rio, R. V. M., Symula, R. E., Wang, J., Lohs, C., Wu, Y., Snyder, A. K., et al. (2012). Insight into the transmission biology and species-specific functional capabilities of tsetse (Diptera: glossinidae) obligate symbiont Wigglesworthia. mBio 3:e0240-11. doi: 10.1128/mBio.00240-11

Seemann, T. (2014). Prokka: rapid prokaryotic genome annotation. Bioinformatics 30, 2068-2069. doi: 10.1093/bioinformatics/btu153

Seshadri, R., Paulsen, I. T., Eisen, J. A., Read, T. D., Nelson, K. E., Nelson, W. C., et al. (2003). Complete genome sequence of the Q-fever pathogen Coxiella burnetii. Proc. Natl. Acad. Sci. U. S. A. 100, 5455-5460. doi: 10.1073/ pnas. 0931379100

Shapiro, M. M., Chakravartty, V., and Cronan, J. E. (2012). Remarkable diversity in the enzymes catalyzing the last step in synthesis of the pimelate moiety of biotin. PLoS One 7:e49440. doi: 10.1371/journal.pone.0049440

Sigognault Flochlay, A., Thomas, E., and Sparagano, O. (2017). Poultry red mite (Dermanyssus gallinae) infestation: a broad impact parasitological disease that still remains a significant challenge for the egg-laying industry in Europe. Parasit. Vectors 10:357. doi: 10.1186/s13071-017-2292-4

Siguier, P., Perochon, J., Lestrade, L., Mahillon, J., and Chandler, M. (2006). ISfinder: the reference centre for bacterial insertion sequences. Nucleic Acids Res. 34, D32-D36. doi: 10.1093/nar/gkj014 
Smith, T. A., Driscoll, T., Gillespie, J. J., and Raghavan, R. (2015). A Coxiellalike endosymbiont is a potential vitamin source for the lone star tick. Genome Biol. Evol. 7, 831-838. doi: 10.1093/gbe/evv016

Toft, C., and Andersson, S. G. E. (2010). Evolutionary microbial genomics: insights into bacterial host adaptation. Nat. Rev. Genet. 11, 465-475. doi: $10.1038 / \mathrm{nrg} 2798$

Tsuchida, T., Koga, R., Fujiwara, A., and Fukatsu, T. (2014). Phenotypic effect of "Candidatus Rickettsiella viridis," a facultative symbiont of the pea aphid (Acyrthosiphon pisum), and its interaction with a coexisting symbiont. Appl. Environ. Microbiol. 80, 525-533. doi: 10.1128/AEM.03049-13

Tsuchida, T., Koga, R., Horikawa, M., Tsunoda, T., Maoka, T., Matsumoto, S., et al. (2010). Symbiotic bacterium modifies aphid body color. Science 330, 1102-1104. doi: 10.1126/science. 1195463

Walker, B. J., Abeel, T., Shea, T., Priest, M., Abouelliel, A., Sakthikumar, S., et al. (2014). Pilon: an integrated tool for comprehensive microbial variant detection and genome assembly improvement. PLoS One 9:e112963. doi: 10.1371/journal.pone.0112963

Wang, Q., Garrity, G. M., Tiedje, J. M., and Cole, J. R. (2007). Naive Bayesian classifier for rapid assignment of rRNA sequences into the new bacterial taxonomy. Appl. Environ. Microbiol. 73, 5261-5267. doi: 10.1128/AEM. 00062-07
Wick, R. R., Schultz, M. B., Zobel, J., and Holt, K. E. (2015). Bandage: interactive visualization of de novo genome assemblies. Bioinformatics 31, 3350-3352. doi: 10.1093/bioinformatics/btv383

Conflict of Interest: The authors declare that the research was conducted in the absence of any commercial or financial relationships that could be construed as a potential conflict of interest.

Publisher's Note: All claims expressed in this article are solely those of the authors and do not necessarily represent those of their affiliated organizations, or those of the publisher, the editors and the reviewers. Any product that may be evaluated in this article, or claim that may be made by its manufacturer, is not guaranteed or endorsed by the publisher.

Copyright (C) 2021 Price, Bartley, Blake, Karp-Tatham, Nunn, Burgess and Nisbet. This is an open-access article distributed under the terms of the Creative Commons Attribution License (CC BY). The use, distribution or reproduction in other forums is permitted, provided the original author(s) and the copyright owner(s) are credited and that the original publication in this journal is cited, in accordance with accepted academic practice. No use, distribution or reproduction is permitted which does not comply with these terms. 